\title{
CLINICAL EVALUATION AND PATIENT SATISFACTION OF SHADE MATCHING BETWEEN NATURAL TEETH AND MONOLITHIC ALL- CERAMIC CROWNS FABRICATED FROM TWO MATERIALS (RANDOMIZED CONTROLLED CLINICAL TRIAL)
}

\author{
Mohamed Bekhiet*, Maha Taymour ${ }^{* *}$ and Mohamed Labib Zamzam ${ }^{* * *}$
}

\begin{abstract}
Aim: The aim of the present study was to assess the shade difference between zirconia strengthened lithium silicate ceramic (Celtra Press) VS lithium disilicate glass ceramics (e.max) in reference to the natural tooth optical properties to determine which monolithic material will replicate the exact shade and optical properties of the corresponding natural tooth structure.

Materials and Methods: 20 teeth esthetic zone requiring full coverage crown -with their adjacent/contralateral are sound and not severely discolored - were selected to receive a ceramic crown and divided into 2 groups:

Group 1: Teeth prepared to receive crowns fabricated from IPS e.max press (Ivoclar Vivadent)

Group 2: Teeth prepared to receive crowns fabricated from Celtra Press (Dentsply Sirona). The patient satisfaction values of the two groups was evaluated using Visual Analogue Scale (VAS) of satisfied or unsatisfied documented in a chart. Color difference in reference to the natural tooth was evaluated by 3 experienced evaluators using modified United States public health service (USPHS) criteria as Alpha(excellent), Bravo (acceptable), Charlie(acceptable but alterations required) and Delta(unacceptable). The color difference $\Delta \mathrm{E}$ (perceptibility threshold) was measured using intraoral spectrophotometer and evaluated for each group.
\end{abstract}

Results: Regarding patient satisfaction, there was no statistically significant difference between (Group I) and (Group II) where ( $\mathrm{p}=1$ ) as both groups showed (100\%) Alpha satisfied. Concerning shade matching, there was no statistically significant difference between (Group I) and (Group II) where $(\mathrm{p}=1)$ as both groups showed (100\%) Alpha.

Conclusions: Within the limitations of this clinical study, Celtra Press and Emax press materials when used for full coverage restorations resulted in excellent patient satisfaction and color matching results. This dictates that both materials have clinical acceptable shade matching and patient satisfaction.

KEYWORDS: Shade matching, patient satisfaction, ceramic crown, full coverage crown, e-max, Celtra Press, (USPHS).

\footnotetext{
* BDS, Faculty of Dentistry, Cairo University.

** Associate Professor of Fixed Prosthodontics, Faculty of Dentistry, Cairo University.

*** Professor of Fixed Prosthodontics, Faculty of Dentistry, Cairo University.
} 


\section{INTRODUCTION}

Media projected perfect appearance affect the society culture and the way of thinking regarding beauty standards which successively led to encouragement of the public to seek esthetic treatments. ${ }^{1}$

Since dental appearance play an important part of facial beauty, placement of a restoration, which enhance the dental appearance, improves the patient's self-esteem and quality of life. Oral health isn't only the absence of oral disease and dysfunction but also its impact on the person's selfconfidence and social life. This is according to the WHO's definition of quality of life. ${ }^{2}$

This increase in patient esthetic demands resulted in the development of number of metal-free fixed prosthesis, as studies showed that metal ceramic restorations have a survival rate of $94 \%$ over 10 years, however, they are usually unable to distribute the reflected light properly, which reduces the final esthetic appearance. ${ }^{2}$

Therefore, all-ceramic restorations including glass ceramics, polycrystalline ceramics and hybrid ceramics were introduced to meet these esthetic requirements. Each type has different physical and optical properties. ${ }^{3}$

Among dental ceramic materials available nowadays is Celtra Press which is a newly introduced zirconia-reinforced lithium silicate ceramic material that consists of a glass matrix and lithium disilicate crystals having a crystal length of about $1.5 \mu \mathrm{m}$ plus nano-scale lithium phosphate. Additionally to Li2O and $\mathrm{SiO} 2$, Celtra Press contains about $10 \%$ zirconia ( $\mathrm{ZrO} 2)$, which is dissolved completely within the glass phase instead of crystalline form. Celtra Press is characterized by a high strength of about 500 MPa and excellent flow properties during pressing. ${ }^{4}$

Nowadays Practitioners face increasing demands and expectations for excellent shade matching as the public pay more attention to esthetics. Thus, excellent shade matching has become an important component of the success of an esthetic restoration. ${ }^{5}$

\section{MATERIALS AND METHODS}

A total of 20 patients ( 10 females, 10 males) were selected for the study with an age range between 20 to 45 years old. Each participant received an allceramic crown for upper tooth in esthetic area (from right first premolar to the left first premolar). The participant's main complaint was to improve their smile. They were informed regarding the alternative treatment options and approved to sign the informed consent before the treatment was done. They were able and willing to maintain good oral hygiene measures. For teeth with substantial loss of tooth structure resulting from caries or endodontically treated, fracture, composite cores (Build-It FR, Pentron clinical. USA) were used along with glassfiber posts-(FibreKleer 4X, Pentron clinical. USA) if required to create the required retention and resistance form. Dental examination, periodontal evaluation, symmetry of gingival level, oral hygiene, dental caries and para-functional habits were assessed. TMJ evaluation was conducted and angle class I participants only were included with proper overbite (cases with very deep bite was excluded from the study). Pre-operative photographs for each patient were taken using $105 \mathrm{~mm}$ Nikon macro lens with twin flash R1C1 mounted on Nikon D7100 DSLR camera (Nikon, Japan.) Figure (1)

Shade was taken using Vita Easyshade V spectrophotometer (VITA, Zahnfabrik, Germany). The value was accepted when two consecutive, identical readings were generated. Shade was also confirmed by VITA 3D-Master shade guide system (VITA, Zahnfabrik, Germany) visually in reference to the contra-lateral/ adjacent tooth under color corrected light (Smile Lite, Smile Line, Switzerland) to avoid metamerism with the assistance of three prosthodontists. Measurements were done in the middle of the tooth in reference to the subject's maxillary corresponding tooth. 


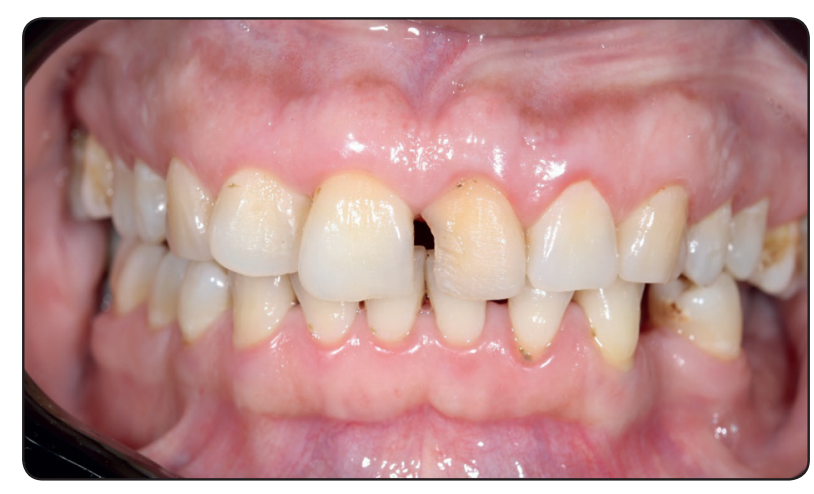

Fig. (1): Preoperative photograph.

A putty silicon was used to obtain an intraoral index for each patient using addition silicon impression material (Panasil, kettenbach, Germany). Each index was vertically cut at the mid of the tooth requiring full-coverage restoration to assess the amount of preparation of incisal, labial and palatal surfaces, respectively. Another putty silicon index was fabricated directly inside the patient mouth with a sectional stock tray which was used later for temporization. Depth grooves were made using two-wheel diamond bur (Komet, Germany) on the incisal edge of the tooth to ensure even reduction of the incisal edge. The grooves made was $1 \mathrm{~mm}$ in diameter which were reduced by tapered diamond stone with round end. Incisal reduction was then increased by the aid of the silicone index to $1.5 \mathrm{~mm}$.A fine tapered diamond stone (Komet, Germany) was used to slice and open the inter-proximal contact. Depth grooves were made on the facial surface using depth cutter wheels (Komet, Germany) in order to ensure even preparation thickness. Final depth of the preparation was marked and accentuated with a pencil. Then the remaining island of the enamel was removed till the depth of original grooves to uniformly reduce the labial surface using a tapered diamond stone with a round end of $1.0 \mathrm{~mm}$ diameter (Komet, Germany). A tapered diamond stone with a round end was used to create chamfer finish line of $1.0 \mathrm{~mm}$ diameter. The chamfer finish line was created supra-gingivally along the free gingival margin. The palatal fossa reduction was performed with diamond football(Komet, Germany) stone and the cingulum was prepared parallel to the long axis of the tooth with a tapered diamond stone with a round end of $1.0 \mathrm{~mm}$ diameter.

The Die shade of the abutment was taken with the IPS Natural Die Material shade guide (IPS Natural Die Material Guide, Ivoclar Vivadent) under color corrected light to produce a working die of similar shade to the underlying tooth substrate to optimize the final result. ${ }^{6}$

Impression procedure was carried out using two step impression technique first putty viscosity (Panasil, kettenbach, Germany) was taken using putty spacer (Kerr, United States) to create even space for the light viscosity silicon. The putty consistency silicon was placed in the tray and inserted in the patient mouth. The tray was removed from the patient mouth after setting of the putty and the spacer was removed, then the light viscosity was applied by using automatic mixing tip and dispensing impression gun which produced complete homogenous mix.

The try-in was performed by using the CAD/ CAM milled castable Polymethyl methacrylate (PMMA) (Yamahachi, Japan) with the final shape of crown. All prepared tooth surfaces were cleaned with polishing brush then washed with water. The CAD/CAM PMMA was initially tried to check marginal fit, shape, contacts, contour and then the overall integration with the lips and finally with the face.

Lithium Disilicate (E-max) and Celtra press ceramics are supplied in the form of ingots to be pressed into full contoured crowns .The PMMA pattern of the proposed restoration was sprued and invested in an IPS Press Vest Premium phosphatebonded material (Ivoclar Vivadent Liechtenstein). Mixing Ratio of IPS Press Vest Premium was 22ml liquid $4 \mathrm{ml}$ distilled water and mixing was done with vacuum Mixer (Renfert twister, Hilzingen, Germany). The investment ring was left to set at 
room temperature for $45 \mathrm{~min}$. Investment ring was placed in the burnout when it was reached $850^{\circ} \mathrm{C}$ and left for $45 \mathrm{~min}$. Following the burnout procedure, the ceramic material (ingot) was pressed into the mould using Ivoclar Programat EP 3010 (Ivoclar Vivadent Liechtenstein), the entry temperature of pressing furnace was at $700^{\circ} \mathrm{C}$ when ring was brought from burnout oven, the temperature for pressing increased to $920^{\circ} \mathrm{C}(100 \mathrm{~g}$ ring $)$ and the hold time before pressing was 25 minutes for lithium disilicate glass ceramics (e.max), and it was 860 ${ }^{\circ} \mathrm{C}(100 \mathrm{~g}$ ring $)$ and the hold time before pressing was 30 minutes for zirconia reinforced lithium silicate ceramics (Celtra press). The mould was removed and allowed to cool down at room temperature. Divesting was done using Glass beads. Fine divestment with polishing beads at 2 bar pressure was also done. The Restoration was then placed in Invex Liquid (Ivoclar Vivadent Liechtenstein) in an ultrasonic bath bath followed by fine divesting using $\mathrm{AL}_{2} \mathrm{O}_{3}$. (This step is for e.max only). Sprue was cut off using diamond disk.

Stain and glaze firing were performed after application of even layer of desired stains (Ivoclar Vivadent / Dentsply Sirona) for characterization directly into the unfired glaze layer using a fine brush . Staining and glazing were performed with the crown seated on the composite die fabricated from IPS Natural Die Material (Ivoclar Vivadent) with the previously determined tooth stump shade in order to serve as the optimum basis for lifelike shade reproduction of the given oral situation. The crowns were fired in a short firing cycle - for 6 minutes at $850^{\circ} \mathrm{C}$ for e.max and for 3 minutes at $750^{\circ} \mathrm{C}$ - according to manufacturer's instructions in the ceramic furnace.

After the crown had been tried in, the internal surface of the e.max press / Celtra Press crown was etched for 20 seconds with $9.5 \%$ buffered hydrofluoric acid (Porcelain etch, Bisco, USA.) then rinsed with water for 20seconds and air dried. $37 \%$ phosphoric acid (Pentron clinical, USA) was then applied for 30 seconds to remove the reaction salts produced by the hydrofluoric acid treatment. The crown then was rinsed with water for 20 seconds then air dried by using three-way syringe following the manufacturer instruction till the crown internal surface appeared clean and had frosty appearance. A single coat of the ceramic primer (Bisco, USA) was then applied to the bonding surface of the crowns and left for 1 minute then air thinned gently.

In order to remove remnants of provisional cements, a prophylaxis paste (DHARMA, USA) and polishing brush (Pro Brush, Kerr,USA) mounted in low speed contra angle was used for cleaning the tooth surfaces prior to bonding. Then, isolation was granted through the use of rubber dam. Selective etching was done to the enamel portion present cervically within the finish line area using 37\% phosphoric acid for 30 seconds followed by rinsing for 20 seconds. Using three-way syringe, the tooth was air-dried, then a fully saturated brush tip of selfetch adhesive bonding agent (Bisco, USA) was used to apply two coats to dentine and light cured for 10 seconds according to the manufacturer instructions. Finally, all teeth surfaces were dried gently for 5 seconds. Luting resin cement (Bisco, USA) with translucent shade was applied to the fitting surfaces of the crown using an auto mixing tip. The crown was placed to the tooth in position till complete seating using finger pressure. Excess cement was removed using silicone rubber tip paint brush to remove excess cement labially and palatally, then a waxed dental floss was used inter-dentally for complete removal of excess cement in between the crown and the adjacent teeth. The crown was then completely light cured from all directions while the margins being covered with oxygen-inhibiting gel (Oxyguard, Panavia, USA.). Moreover, an articulating paper (Bausch USA) was used to check for any occlusal interferences after complete curing. Figure (2) 


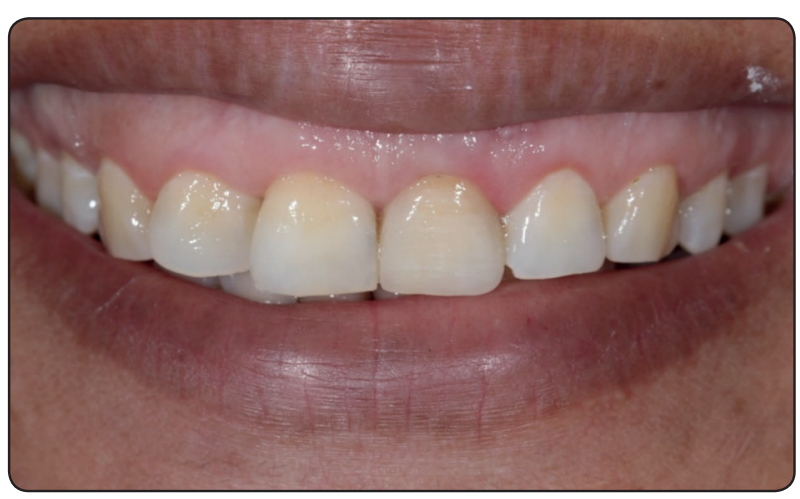

Fig. (2): After cementation of the crown.

The shade matching (Primary outcome) of the two groups was assessed with the help of three experienced evaluators using modified United States Public Health Service (USPHS) criteria as follow: Alpha: excellent match, Bravo:less than ideal but no modification required,Charlie: acceptable and shade modification required, Delta : unacceptable shade match.

The patient satisfaction outcome (secondary outcome) of the two groups was assessed by using Visual Analogue Scale (VAS) which is binary and documented in chart in the form of satisfied and unsatisfied. The patients were given a handheld mirror to view their teeth and restoration and choose whether the are satisfied with the definitive restoration or unsatisfied.

The color difference between the two groups was assessed intraorally by the mean of spectrophotometer (Vita Easyshade V) in accordance to the contralateral / adjacent tooth. The Data obtained were recorded, tabulated and statistically analyzed.

\section{RESULTS}

The results were analyzed by Graph Pad Instat (Graph Pad, Inc.) software for windows.

A value of $\mathrm{P} \leq 0.05$ was considered statistically significant. The obtained data from the assessment criteria evaluated for each group were statistically analyzed using the Chi-square test that was performed in categorical data.
Sample size $(\mathrm{n}=10)$ was large enough to detect large effect size for main effects and pair wise comparison with satisfactory level of power of $80 \%$ and a $95 \%$ confidence level.

Shade match showed statistically non-significant results as majority of the patients stated Alpha score $80 \%$ while minority showed Bravo score $20 \%$ with $0 \%$ for, Charlie and Delta scores for both groups. This was statistically non-significant as proved by chi-square test $(\mathrm{p}=1>0.05)$

TABLE (1) frequent distribution (\%) of Shade match with aesthetics associated with restoration for both groups

\begin{tabular}{|c|c|c|c|c|c|}
\hline \multirow{2}{*}{ Variable } & Parameter & \multicolumn{4}{|c|}{ Shade match } \\
\cline { 2 - 6 } & Outcome & Alpha & Bravo & Charlie & Delta \\
\hline \multirow{2}{*}{$\begin{array}{c}\text { Material } \\
\text { group }\end{array}$} & e.max Press & $8(80 \%)$ & $2(20 \%)$ & $0(0 \%)$ & $0(0 \%)$ \\
\cline { 2 - 6 } & Celtra Press & $8(80 \%)$ & $2(20 \%)$ & $0(0 \%)$ & $0(0 \%)$ \\
\hline \multirow{2}{*}{$\begin{array}{c}\text { Chi square } \\
\text { test }\end{array}$} & Chi value & \multicolumn{4}{|c|}{0} \\
\cline { 2 - 6 } & P value & \multicolumn{4}{|c|}{$1 \mathrm{~ns}$} \\
\hline
\end{tabular}

Ns; non-significant $(p>0.05)$

Patient satisfaction showed statistically nonsignificant results as $100 \%$ of the patients were satisfied while $0 \%$ were dissatisfied by the restoration in both groups. This was statistically non-significant as proved by chi-square test $(\mathrm{p}=1>0.05)$

TABLE (2) frequent distribution (\%) of Patient's satisfaction with aesthetics associated with restoration for both groups

\begin{tabular}{|c|c|c|c|}
\hline \multirow{2}{*}{} & Parameter & \multicolumn{2}{|c|}{ Patient satisfaction } \\
\cline { 2 - 4 } & Outcome & Satisfied & Dissatisfied \\
\hline \multirow{2}{*}{$\begin{array}{c}\text { Material } \\
\text { group }\end{array}$} & e.max Press & $10(100 \%)$ & $0(0 \%)$ \\
\cline { 2 - 4 } & Celtra Press & $10(100 \%)$ & $0(0 \%)$ \\
\hline $\begin{array}{c}\text { Chi } \\
\text { square } \\
\text { test }\end{array}$ & Chi value & \multicolumn{2}{|c|}{0} \\
\cline { 2 - 4 } & P value & \multicolumn{2}{|c|}{$1 \mathrm{~ns}$} \\
\hline
\end{tabular}

Ns; non-significant $(p>0.05)$ 
TABLE (3) Comparison of color changes test results (Mean \pm SD) between both groups

\begin{tabular}{|c|c|c|c|c|c|c|c|}
\hline \multirow{2}{*}{\multicolumn{2}{|c|}{ Variables }} & \multirow{3}{*}{$\begin{array}{c}\text { Mean } \\
1.12\end{array}$} & \multirow{3}{*}{$\begin{array}{c}\boldsymbol{S D} \\
0.41\end{array}$} & \multicolumn{2}{|c|}{$95 \% C I$} & \multicolumn{2}{|c|}{ Range } \\
\hline & & & & \multirow{2}{*}{$\begin{array}{c}\text { Lower } \\
0.83\end{array}$} & \multirow{2}{*}{$\begin{array}{c}\text { Upper } \\
1.42\end{array}$} & \multirow{2}{*}{$\frac{\text { Mini. }}{0.40}$} & \multirow{2}{*}{$\begin{array}{r}\text { Maxi. } \\
1.60\end{array}$} \\
\hline \multirow{2}{*}{ Material group } & e.max Press & & & & & & \\
\hline & Celtra Press & 1.15 & 0.66 & 0.68 & 1.62 & 0.40 & 2.50 \\
\hline \multirow{2}{*}{ t-test } & t-value & \multicolumn{6}{|c|}{0.12} \\
\hline & P value & \multicolumn{6}{|c|}{$0.9044 \mathrm{~ns}$} \\
\hline
\end{tabular}

* Significant $(p<0.05) \quad n s ;$ non-significant $(p>0.05)$

Color changes It was found that Celtra Press group recorded statistically non-significant higher color changes than e.max Press group as indicated by student $t$-test $(\mathrm{t}=0.12, \mathrm{P}=0.9044>0.05)$ as the mean \pm SD values of color changes recorded for e.max Press group were $(1.12 \pm 0.41 \Delta \mathrm{E})$ meanwhile the mean \pm SD value recorded with Celtra Press group $(1.15 \pm 0.66 \Delta \mathrm{E})$.

\section{DISCUSSION}

In the presented study all-ceramic crowns were made from two different ceramic materials: IPS e.max Press (lithium disilicate based glass ceramics) and Celtra Press (zirconia strengthened lithium silicate ceramics (ZLS). IPS e.max is considered one of the most esthetic ceramic materials due to the needle shaped lithium disilicate crystals distributed within the glassy matrix that reduces the scattering of the incident light. In addition to its characteristic chameleon effect. ${ }^{3}$ Celtra Press (zirconia strengthened lithium silicate ceramics) is a lithium silicate ceramic that is characterized by the presence of $10 \%$ zirconia particles completely dissolved within its matrix, this combination provides the material with high flexure strength and excellent regarding the translucency and opalescence. ${ }^{7}$

Vita Easyshade V spectrophotometer was used for shade matching in reference to Chen et al., $2012{ }^{8}$ who suggested based on his systematic review that instrumental method provides the most precise and accurate shade-matching outcomes. Moreover N.Alghazali et al., $2006^{9}$ assessed the performance of Vita Easyshade spectrophotometer on the esthetic dental restorations, and concluded that Easy Shade spectrophotometer is a reliable precise instrument in color measurements of resin-based composites and porcelain materials.

Visual shade selection using shade guides was used as a confirmatory method with digital shade selection via spectrophotometer. Vitapan 3D-Master shade guide was used in this study due to its common use in dental clinics. Zenthöfer et al., $2014^{10}$ suggested the use of VITA 3D MASTER shade guide as it provided superior matching results, it has high reliability than that of Vita Classical among most clinicians.

Conventional all ceramic full coverage preparation was performed by preparing the tooth following manufacturer's recommendations for all-ceramic crown. ${ }^{11,12}$ In order to standardize the preparation design silicon index was fabricated for checking the final amount of reduction for the teeth, then twowheel depth cutter was used to perform optimum depth cutting. This procedure ensured uniform reduction. ${ }^{13}$

Pressing technique were used in this study for both materials since it is very popular in the laboratory due to ease of use and the resultant restoration is of high fracture toughness and excellent marginal fit. ${ }^{14}$ 
In the present study, according to Magne et al., $2005^{15}$ remnants of provisional cement were removed by a Prophylaxis paste and polishing brush mounted in low speed contra angle to ensure that the tooth surface is completely cleaned from provisional cement and contaminations which might affect the bond strength. The use of a clear glycerin which is water soluble gel as an optical fluid to help predict the influence of resin cement shade on the definitive restoration. ${ }^{16}$

The Alpha score of no color change was recorded for majority of the full-coverage restorations. Additionally, there was no statistically significant difference between the two groups regarding the color change. This might be related to the strict protocol followed for shade matching in addition to the similarity of the microstructure of both materials IPS e.max press and Celtra Press being glass based ceramics .Studies supporting our results that done by Fasbinder et al., 2014 ${ }^{17}$ who stated that Alpha scores were noted for 2 years of service lithium disilicate crowns which supports the results acquired from the subjects of this study. As for the zirconia reinforced lithium silicate ceramics, Rinke et al., $2015^{18}$ found that the high esthetic properties of zirconia reinforced lithium silicate ceramics (ZLS) may be due to the homogenous microstructure consisting of very fine lithium disilicate and lithium metasilicate crystals with average size of 0.5-0.7 $\mu \mathrm{m}$ which are 4 to 8 times smaller than lithium disilicate crystals. Yet Chaiyabutr et al., $2011^{19}$ found that there is color difference of glass-ceramic lithium disilicate-reinforced ceramics and suggested that this might be due to the optical properties of the ceramic itself that may be affected by the color of the underlying tooth structure and in turn affect the final color of the restoration.
All participants were satisfied with their final restorations. Moreover, there was statistically nonsignificant difference between the results of the two groups. These results might be reasonable due to the strict protocol followed during shade selection process and the alpha score of ideal shade matching obtained by the prosthodontists evaluators..$^{20}$ stated that patient reported superior satisfaction results which may have been affected by the light shade of the restoration. Contradicting to our study results, a study by Shah et al., $2014^{21}$ who found that the overall patient satisfaction rating were moderate and suggested that the level of patient education may have affected the final results as participants with secondary and tertiary level of education were more satisfied with the color of their final restoration compared to participants with primary level of education.

More clinical studies are required with prolonged follow-up periods in order to evaluate long-term esthetic clinical performance of the materials along with patient satisfaction in order to be used in different situations for better esthetic outcome. Other clinical situations as with patients with severely discolored teeth and different processing techniques as layering or cut-back might affect the final esthetic outcome.

\section{CONCLUSIONS}

Within the limitations of this clinical study, Celtra Press and e.max press full coverage restoration provided excellent patient satisfaction and color matching to adjacent / contralateral natural tooth. This indicates that both materials have clinical acceptable shade matching and patient satisfaction and can be recommended to be used for esthetic anterior restorations with high predictable esthetic outcome. 


\section{REFRENCES}

1. Tin-Oo MM, Saddki N, Hassan N. Factors influencing patient satisfaction with dental appearance and treatments they desire to improve aesthetics. BMC Oral Health. 2011;11(1). doi:10.1186/1472-6831-11-6

2. Samorodnitzky-Naveh GR, Geiger SB, Levin L. Patients' satisfaction with dental esthetics. J Am Dent Assoc. 2007;138(6):805-808. doi:10.14219/jada.archive.2007.0269

3. Bajraktarova-Valjakova E, Korunoska-Stevkovska V, Kapusevska B, Gigovski N, Bajraktarova-Misevska C, Grozdanov A. Contemporary dental ceramic materials, a review: Chemical composition, physical and mechanical properties, indications for use. Open Access Maced J Med Sci. 2018;6(9):1742-1755. doi:10.3889/oamjms.2018.378

4. Press C. FactFile FactFile. :1-8.

5. Ishikawa-Nagai S, Yoshida A, Sakai M, Kristiansen J, Da Silva JD. Clinical evaluation of perceptibility of color differences between natural teeth and all-ceramic crowns. J Dent. 2009;37(SUPPL. 1):57-63. doi:10.1016/j. jdent.2009.04.004

6. Chu SJ, Mieleszko AJ. Color-matching strategies for non-vital discolored teeth: Part 1. Laboratory ceramic veneer fabrication solutions. J Esthet Restor Dent. 2014;26(4):240-246. doi:10.1111/jerd.12111

7. TRAINI T, SINJARI B, PASCETTA R, et al. The zirconiareinforced lithium silicate ceramic: lights and shadows of a new material. Dent Mater J. 2016;35(5):748-755. doi:10.4012/dmj.2016-041

8. Bagis B, Turgut S. Optical properties of current ceramics systems for laminate veneers. J Dent. 2013;41(SUPPL. 3). doi:10.1016/j.jdent.2012.11.013

9. Khashayar G, Bain PA, Salari S, Dozic A, Kleverlaan CJ, Feilzer AJ. Perceptibility and acceptability thresholds for colour differences in dentistry. J Dent. 2014;42(6):637644. doi:10.1016/j.jdent.2013.11.017

10. Zenthöfer A, Wiesberg S, Hildenbrandt A, Reinelt G, Rammelsberg P, Hassel AJ. Selecting VITA classical shades with the VITA 3D-master shade guide. Int J Prosthodont. 27(4):376-382. doi:10.11607/ijp.3770

11. Carsten DL. Successful shade matching--what does it take? Compend Contin Educ Dent. 2003;24(3).
12. Kim-Pusateri S, Brewer JD, Davis EL, Wee AG. Reliability and accuracy of four dental shade-matching devices. J Prosthet Dent. 2009;101(3):193-199. doi:10.1016/S00223913(09)60028-7

13. Ram HK, Shah RJ, Agrawal HS. Evaluation of three different tooth preparation techniques for metal ceramic crowns by comparing preparation depths: An in vitro study. J Indian Prosthodont Soc. 2015;15(2):162-167. doi:10.4103/0972-4052.159961

14. Denry I. How and when does fabrication damage adversely affect the clinical performance of ceramic restorations? Dent Mater. 2013;29(1):85-96. doi:10.1016/j.dental.2012.07.001

15. Magne P, Kim TH, Cascione D, Donovan TE. Immediate dentin sealing improves bond strength of indirect restorations. J Prosthet Dent. 2005;94(6):511-519. doi:10.1016/j. prosdent.2005.10.010

16. Jirajariyavej B, Wanapirom P, Anunmana C. Influence of implant abutment material and ceramic thickness on optical properties. J Prosthet Dent. 2018;119(5):819-825. doi:10.1016/j.prosdent.2017.05.015

17. Fasbinder DJ, Dennison JB, Heys D, Neiva G. A Clinical Evaluation of Chairside Lithium Disilicate CAD/ CAM Crowns. J Am Dent Assoc. 2010;141:10S-14S. doi:10.14219/jada.archive.2010.0355

18. Rinke S, Rödiger M, Ziebolz D, Schmidt AK. Fabrication of Zirconia-Reinforced Lithium Silicate Ceramic Restorations Using a Complete Digital Workflow. Case Rep Dent. 2015;2015. doi:10.1155/2015/162178

19. Chaiyabutr Y, Kois JC, LeBeau D, Nunokawa G. Effect of abutment tooth color, cement color, and ceramic thickness on the resulting optical color of a CAD/CAM glass-ceramic lithium disilicate-reinforced crown. J Prosthet Dent. 2011;105(2):83-90. doi:10.1016/S0022-3913(11)60004-8

20. Ballard E, Metz MJ, Harris BT, et al. Satisfaction of dental students, faculty, and patients with tooth shade-matching using a spectrophotometer. J Dent Educ. 2017;81(5):545553. doi:10.21815/JDE.016.022

21. J Shah DR, G Malek DF, Agarwal DP. A Study of Patient Satisfaction with Maxillary Anterior Teeth Restorations and Desirable Esthetic Treatment Options. IOSR J Dent Med Sci. 2014;13(10):79-86. doi:10.9790/0853131037986 